\title{
The application of CYFRA21-1 in cervical lesions screening in high-risk human papillomavirus infected women
}

\author{
Feng Yang-chun, Yang Jia, Liu Cheng-ming, Huang Yan-chun \\ The Clinical Laboratory Center, Tumor Hospital Affiliated to Xinjiang Medical University, Urumqi, People's Republic of China
}

\begin{abstract}
Objectives: To investigate the role of cytokeratin-19 fragment (CYFRA21-1) in cervical lesions screening in high-risk human papillomavirus (HR-HPV) infected women.

Material and methods: The study was a retrospective study. First, the results of CYFRA21-1, cytology (TCT), and HR-HPV examinations of 1039 outpatients from gynecology department in Tumor Hospital Affiliated to Xinjiang Medical University were collected. Then, the data was analyzed using a series of statistical methods.

Results: There was a correlation between CYFRA21-1 levels and HPV-DNA load in HR-HPV infected women $\left(r_{s}=0.711\right.$, $p=0.015$ ). CYFRA21-1 levels and positive rate increased along with deepening of cervical cell lesions. In HR-HPV infected women, there was a statistically significant difference $(t=6.022, p<0.001)$ in CYFRA21-1 levels between the group with cytological lesions $(4.87 \pm 1.58 \mathrm{ng} / \mathrm{mL}$ ) and the group with normal cytology $(2.52 \pm 0.96 \mathrm{ng} / \mathrm{mL})$. Positive rates of $\mathrm{CY}$ FRA21-1 in the two groups were $62.06 \%$ and $7.83 \%$, respectively, and also exhibited statistically significant differences $\left(x^{2}=74.624, p<0.001\right)$. When diagnosing cytological lesions via CYFRA21-1 in HR-HPV infected women, sensitivity was $62.07 \%$, specificity was $92.17 \%$, positive predictive value was $88.89 \%$, negative predictive value was $70.67 \%$. Compared to CYFRA21-1 negative women, cytological lesions were detected 19.273 times more often in CYFRA21-1 positive women.

Conclusion: CYFRA21-1 could provide a reference idea for further diagnosis of women who are infected with HR-HPV but whose cytology is normal.
\end{abstract}

Key words: high-risk, human papillomavirus, CYFRA21-1, cervical lesions

\section{INTRODUCTION}

Human papillomavirus (HPV) is a type of virus which tends to parasitize epithelial cells. A HPV infection could lead to hyperplasia in a variety of squamous epithelial tissues, such as cervical squamous epithelial cells, esophageal squamous cells and so on [1-3]. CYFRA21-1 widely exists in layered and squamous epithelial cells and, when a malignant hyperplasia occurs in squamous cells, the levels of serum CYFRA21-1 rise [4]. Cervical malignant lesions or cervical cancer are caused by a persistent infection with high-risk HPV (HR-HPV) which leads to immortalized hyperplasia of cervical epithelial cells [5]. HR-HPV includes hpv16, hpv18, hpv31, hpv33, hpv35, hpv45, hpv51, hpv52, hpv56, hpv58, hpv61 and so on. $90 \%$ 95\% of cervical cancer is squamous cell carcinoma, so the goal is to research the relationship between the pathological changes in exfoliated cervical cells and CYFRA21-1 in HR-HPV infected women and thus to help to shunt screen HR-HPV infected women in the gynecology clinic.

\section{MATERIAL AND METHODS}

\section{The source of the sample}

The data was collected from outpatients who visited the gynecology clinic in Tumor Hospital Affiliated to Xinjiang Medical University between July $1^{\text {st }}, 2011$ and July $31^{\text {st }}$, 2014. The results of their CYFRA21-1, HR-HPV-DNA and Thinprep Cytology Test (TCT, a liquid-based cytology method) 
were obtained through the hospital laboratory information system. The data of those outpatients whose TCT result indicated adenocarcinoma was excluded. Ultimately, data of 1039 outpatients, aged between 18 years old and 71 years old, with a median age of 47 years old, was collected.

\section{Detection methods}

HR-HPV test used the Digene HPV Test kit (Digene Corporation, USA) and related instruments (9700 GeneAmp PCR System etc.), and its principle was to generate results using the hybrid capture II method (HCII). The result of $\mathrm{HCll}$ was expressed via RLU value, the value of relative light units/cutoff value (threshold). RLU value represented the HR-HPV-DNA load in cervical epithelial cells. A RLU value greater than 1.0 was deemed to indicate positive HR-HPV infection. CYFRA21-1 was detected using Abbott i2000 automatic micro particle chemiluminescence analyzer and original kits. Reference interval for CYFRA21-1 levels was $0 \sim 3.3 \mathrm{ng} / \mathrm{mL}$; a value greater than $3.3 \mathrm{ng} / \mathrm{mL}$ was considered to be a positive result for CYFRA21-1. TCT results were expressed through terms used in cervical cytological diagnosis of TBS classification system, including Within Normal Limits (WNL), Atypical Squamous Cells (ASC), Low-grade Squamous Intraepithelial Lesions (LSIL), High-grade Squamous Intraepithelial Lesion (HSIL) and Squamous Cell Carcinoma (SCC). Positive cytological diagnosis included any lesions classified as ASC or above.

\section{Statistical methods}

Statistical analyses were performed using the SPSS statistics software version 17.0. Data was presented as $\bar{x} \pm$ SD, median (M) and quarterback spacing (Q). Quantitative variables of two independent groups were compared using the parametric Student t-test. Spearman rank correlation analysis was used for assessing the correlation between the two indexes. Quantitative variables of many independent groups were compared using one-way analysis of variance (ANOVA) and if the difference in results was statistically significant, then the Dunnett-T3 method was used to compare every pair of groups. Positive rates between different groups were compared using $\chi^{2}$ test. Significance level was $a=0.05$, $\mathrm{p}<0.05$ indicated statistical significance.

\section{RESULTS}

\section{General data statistics}

Out of 1039 cases which formed the sample, there were 231 cases of women testing positively for HR-HPV infection; the positive rate was $22.91 \%$. In 87 cases CYFRA21-1 tests indicated positive results; the positive rate was $8.37 \%$. In 121 cases TCT results were positive; the positive rate was $12.22 \%$.
Table 1. The comparison of CYFRA21-1 levels and positive rates between different cervical lesion groups

\begin{tabular}{|l|c|c|c|}
\hline \multirow{2}{*}{ Group } & N & \multicolumn{2}{|c|}{ CYFRA21-1 } \\
\cline { 2 - 4 } & & $\begin{array}{c}\text { Levels } \\
{[\mathrm{ng} / \mathrm{mL}]}\end{array}$ & $\begin{array}{c}\text { Positive rate } \\
(\%)\end{array}$ \\
\hline Negative group & 912 & $1.36 \pm 0.54$ & 1.64 \\
\hline Low lesion group & 61 & $2.79 \pm 0.79$ & 37.71 \\
\hline Moderate lesion group & 40 & $5.05 \pm 1.38$ & 60.00 \\
\hline Severe lesion group & 26 & $9.86 \pm 0.82$ & 96.15 \\
\hline
\end{tabular}

\section{The correlation between HR-HPV-DNA load and CYFRA21-1}

CYFRA21- 1 levels $(2.79 \pm 0.82 \mathrm{ng} / \mathrm{mL})$ in HR-HPV positive cases showed a statistically significant difference ( $t=5.014, p<0.001$ ) compared to HR-HPV negative cases $(1.36 \pm 0.51 \mathrm{ng} / \mathrm{mL})$. Due to the fact that HPV-DNA quantitative variables showed skewness in distribution in HR-HPV infected cases, they are presented using the median (M) and quarterback spacing $(Q)$, which are 5.78 and 42.37 , respectively. In Spearman rank correlation analysis, HPV-DNA load showed correlation with CYFRA21-1 $\left(r_{s}=0.711, p=0.015\right)$.

\section{The difference in CYFRA21-1 levels and positive rates between different cervical cell lesions groups}

Based on TCT results, 1039 cases which formed the sample were divided into four groups: negative group (912 cases) including WNL women, Low lesion group (61 cases) including ASC and LSIL women, Moderate lesion group (40 cases) including HSIL women and Severe lesion group (26 cases) including SCC women. CYFRA21-1 test results for all four groups are shown in Table 1. ANOVA $(F=47.382$, $\mathrm{p}<0.001$ ) showed statistically significant differences in $\mathrm{CY}$ FRA21-1 levels among the four groups; Dunnett-T3 method was subsequently employed and showed that statistical differences existed between any two groups ( $p<0.001)$. CYFRA21-1 positive rates in the four groups also showed statistical significance $\left(X^{2}=522.300, p<0.001\right)$.

\section{The correlation between cervical lesions and CYFRA21-1 in HR-HPV infected women}

Of 231 patients with HR-HPV infection, 115 cases showed no cytological pathological changes. CYFRA21-1 was com-

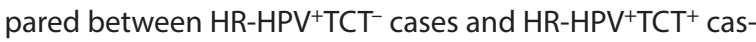
es. Comparative results are shown in Table 2.

\section{CYFRA21-1 used to diagnose cervical cell lesions in HR-HPV infected cases}

When diagnosing cervical cell lesions in HR-HPV infected women as shown in Table 3, sensitivity was $62.07 \%$, 


\begin{tabular}{l} 
Table 2. The expression of CYFRA21-1 in different HR-HPV infected \\
cases \\
\hline
\end{tabular}

${ }^{*} t=6.022, p<0.001 ; *{ }^{*} X^{2}=74.624, p<0.001$; both showed statistically significant differences

Table 3. The values of CYFRA21-1 used to diagnose cervical cell lesions in HR-HPV infected cases

\begin{tabular}{|l|c|c|}
\hline & TCT $^{+}$ & TCT \\
\hline HR-HPV+CYFRA21-1 $^{+}$ & 72 & 9 \\
\hline HR-HPV+CYFRA21-1- $^{-}$ & 44 & 106 \\
\hline
\end{tabular}

specificity was $92.17 \%$, positive predictive value was $88.89 \%$, negative predictive value was $70.67 \%$. The odds ratio (OR) of diagnosing cervical cell lesions in positive $C Y$ FRA21-1 cases compared to negative CYFRA21-1 cases was 19.273; 95\% Cl was 8.861-41.917. It means that cervical cell lesions are 19.273 times more likely to appear in positive CYFRA21-1 cases than in negative CYFRA21-1 cases.

\section{DISCUSSION}

Harald zur Hauser proved that HR-HPV infection is a high risk factor of squamous cell carcinomas in the 1970s [6]. Recent studies have shown that persistent HR-HPV infections are closely related to malignant lesions of cervical epithelial cells. Therefore, HR-HPV test has been incorporated into the process of screening for cervical lesions in women. Currently, the most widely used clinical screening system for cervical lesions in women in the gynecology clinic is HPV testing in combination with TCT examination. CYFRA21-1 is a common serum tumor marker of squamous cell carcinoma which has a certain significance in auxiliary diagnosis of cervix lesions [7-10]. In this study, during the statistical analysis of gynecology clinic outpatients, several observations were made. First, it was found that CYFRA21-1 levels exhibit obvious differences between HR-HPV positive and HR-HPV negative populations. Next, it was discovered that a clear positive correlation $\left(r_{s}=0.711\right)$ exists between HR-HPV-DNA and CYFRA21-1 in HR-HPV infected population. It means that HR-HPV infection could affect CYFRA21-1 levels; therefore, CYFRA21-1 could help in shunt screening for cervical malignant lesions in HR-HPV infected patients in the gynecology clinic.

In 127 cases of TCT positive patients, the study found that the levels and positive rates of CYFRA21-1 have been increasing following deepening of cervical lesions, which is consistent with previous reports [11]. Because CYFRA21-1 as a serum tumor marker is not as closely related to cervical lesions as HR-HPV-DNA, in the low-grade and moderate lesion groups there were some negative CYFRA21-1 cases. To sum up, in HR-HPV infected women, CYFRA21-1 and HR-HPV virus load had good correlation, and the serum concentration of CYFRA21-1 increased along with deepening of cervical lesions. It could therefore be used in auxiliary diagnosis of cervical lesion index for suspicious cervical lesions; positive CYFRA21-1 could also prompt further diagnosis of cervical lesions.

Due to the fact that HR-HPV testing combined with TCT is the most commonly used screening method [12-16], the question of how to further shunt screening is a common problem in case of HR-HPV positive but TCT negative patients. Clinicians usually consider HR-HPV positive patients to have suspected cervical lesions, so the difference in expression of CYFRA21-1 between $\mathrm{HPV}^{+} \mathrm{TCT}^{+}$and $\mathrm{HPV}^{+} \mathrm{TCT}^{-}$groups is very significative. This study found that the levels and positive rates of CYFRA21-1 show significant differences between $\mathrm{HPV}^{+} \mathrm{TCT}^{+}$and $\mathrm{HPV}^{+} \mathrm{TCT}^{-}$patients.

For further study, diagnosing cervical lesions using $\mathrm{CY}$ FRA21-1 in HR-HPV infected women had good specificity, equal to $92.17 \%$. Previous reports suggest that CYFRA21-1 as a tumor marker has high sensitivity and low specificity in diagnosis of HPV infected cervical cancer [17], which does not match the results of this study. This inconsistency may be the result of different research objects: the object of previous reports was HPV infected cervical cancer, but the object of this study were HR-HPV infected cervical lesions in patients.

This study also found that appearance of cervical lesions in positive CYFRA21-1 cases was 19.273 times more likely compared to negative CYFRA21-1 cases in all HR-HPV infected women. Results of further CYFRA21-1 tests for HR-HPV infected patients indicating positive CYFRA21-1 denote two possibilities: first, TCT may have a false negative cytology, which prompts the doctor to reexamine TCT or perform further cytology checks; second, positive CYFRA21-1 means that occurrence of cervical cell lesions in the future is much more likely, which requires patients to shorten the interval between follow-up cytological examinations in order to quickly discover any future cervical cell lesions. Conversely, if CYFRA21-1 is in the normal range or at a very low level, the interval between follow-up examinations could be appropriately relaxed in the future, reducing economic burden for the patients.

\section{CONCLUSIONS}

There was certain clinical significance of CYFRA21-1 for shunting to screen cervical lesions in HR-HPV infected women. However, the study only encompassed 238 cases of HR-HPV infected patients; the sample number was small. Also, the positive cutoff value of CYFRA21-1 in the study was taken from the testing laboratory reference range; a more 
detailed study should be performed to obtain a more significant cutoff value of CYFRA21-1 for shunt screening the HR-HPV infected population.

\section{REFERENCES}

1. Xi R, Zhang X, Chen X, [et al.]. Human papillomavirus 16 infection predicts poor outcome in patients with esophageal squamous cell carcinoma. Onco Targets Ther. 2015, 8, 573-581.

2. Schettino MT, Ammaturo FP, Grimaldi E, [et al.]. Persistent papillomavirus type-31 and type-45 infections predict the progression to squamous intraepithelial lesion. Taiwan J Obstet Gynecol. 2014, 53, 494-497.

3. Feng YC, Yang J, Liu CM, [et al.]. DNA ploidy of cervical epithelial cells should be a cure criterion of high-risk HPV infection in Xinjiang Uygur women. Onco Targets Ther. 2015, 8, 827-833.

4. Wang YX, Hu D, Yan X. Diagnostic accuracy of Cyfra 21-1 for head and neck squamous cell carcinoma:a meta-analysis. Eur Rev Med Pharmacol Sci. 2013, 17, 2383-2389.

5. Li C, Ma C, Zhang W, [et al.]. The immune function differences and high-risk human papillomavirus infection in the progress of cervical cancer. Eur J Gynaecol Oncol. 2014, 35, 557-561.

6. zur Hauser $\mathrm{H}$. Human papillomaviruses and their possible role in squamous cell carcinomas. Curr Top Microbiol Immunol. 1977,78:1-30.

7. Chmura A, Wojcieszek A, Mrochem J, [et al.]. Usefulness of the SCC, CEA, CYFRA 21.1, and CRP markers for the diagnosis and monitoring of cervical squamous cell carcinoma. Ginekol Pol. 2009, 80, 361-366.
8. Bedkowska GE, Ławicki S, Szmitkowski M. Molecular markers of carcinogenesis in the diagnostics of cervical cancer. Postepy Hig Med Dosw (Online). 2009, 27, 99-105.

9. Davelaar EM, van de Lande J, von Mensdorff-Pouilly S, [et al.]. A combination of serum tumor markers identifies high-risk patients with early-stage squamous cervical cancer. Tumour Biol. 2008, 29, 9-17.

10. Markowska J.Tumor makrers in cervical cancer. Ginekol Pol. 2007, 78,715-718.

11. $\mathrm{Ni} \mathrm{FH}, \mathrm{Yu} \mathrm{CS}$. The clinical value of tumor markers detection in various kinds of HPV infected cervical disease. J Radioimmunol. 2013, 26, 703-704.

12. Yang $L, H e Z$, Huang $X Y$, [et al.]. Prevalence of human papillomavirus and the correlation of HPV infection with cervical disease in Weihai, China. Eur J Gynaecol Oncol. 2015, 36, 73-77.

13. Zhou H, Jia Y, Shen J, [et al.]. Gynecologic infections seen in ThinPrep cytological test in Wuhan, China. Front Med. 2014 , 8, 236-240.

14. Wang JL, Yang YZ, Dong WW, [et al]. Application of human papillomavirus in screening for cervical cancer and precancerous lesions. Asian Pac J Cancer Prev. 2013, 14, 2979-2982.

15. Lin M, Yang LY, Li LJ, [et al.]. Genital human papillomavirus screening by gene chip in Chinese women of Guangdong province. Aust N Z J Obstet Gynaecol. 2008, 48, 189-194.

16. Zhang R, Shi TY, Ren Y, [et al.]. Risk factors for human papillomavirus infection in Shanghai suburbs: a population-based study with 10,000 women. J Clin Virol. 2013, 58, 144-148.

17. Qian QQ, Ding HY, Zhou W. Analysis of serum tumor markers in patients with different types of HPV infected cervical disease. Chinese J Health Lab Technol. 2013, 23, 1208-1209. 